\title{
Is there a highly magnetized neutron star in GX 301-2?
}

\author{
V. Doroshenko ${ }^{1}$, A. Santangelo ${ }^{1}$, V. Suleimanov ${ }^{1,5}$, I. Kreykenbohm ${ }^{2,3}$, R. Staubert ${ }^{1}$, C. Ferrigno ${ }^{1,4}$, and D. Klochkov ${ }^{1}$ \\ 1 Institut für Astronomie und Astrophysik, Sand 1, 72076 Tübingen, Germany \\ e-mail: doroshv@astro.uni-tuebingen.de \\ 2 Dr. Karl Remeis-Sternwarte, Sternwartstrasse 7, 96049 Bamberg, Germany \\ 3 Erlangen Centre for Astroparticle Physics (ECAP), Erwin-Rommel-Strasse 1, 91058 Erlangen, Germany \\ 4 ISDC Data Centre for Astrophysics, Chemin d'Écogia 16, 1290 Versoix, Switzerland \\ 5 Kazan State University, Kremlevskaya 18, 420008, Kazan, Russia
}

Received 21 July 2009 / Accepted 17 February 2010

\section{ABSTRACT}

\begin{abstract}
We present the results of an in-depth study of the long-period X-ray pulsar GX 301-2. Using archival data of INTEGRAL, RXTE $A S M$, and CGRO BATSE, we study the spectral and timing properties of the source. Comparison of our timing results with previously published work reveals a secular decay of the orbital period at a rate of $\simeq-3.25 \times 10^{-5} \mathrm{~d} \mathrm{yr}^{-1}$, which is an order of magnitude faster than for other known systems. We argue that this is probably result either of the apsidal motion or of gravitational coupling of the matter lost by the optical companion with the neutron star, although current observations do not allow us to distinguish between those possibilities. We also propose a model to explain the observed long pulse period. We find that a very strong magnetic field $B \sim 10^{14} \mathrm{G}$ can explain the observed pulse period in the framework of existing models for torques affecting the neutron star. We show that the apparent contradiction with the magnetic field strength $B_{\mathrm{CRSF}} \sim 4 \times 10^{12} \mathrm{G}$ derived from the observed cyclotron line position may be resolved if the line formation region resides in a tall accretion column of height $\sim 2.5-3 R_{\mathrm{NS}}$. The color temperature measured from the spectrum suggests that such a column may indeed be present, and our estimates show that its height is sufficient to explain the observed cyclotron line position.
\end{abstract}

Key words. pulsars: individual: GX 301-2 - stars: neutron - binaries: general

\section{Introduction}

GX 301-2 (also known as 4U 1223-62) is a high-mass X-ray binary system, consisting of a neutron star orbiting the early B-type optical companion Wray 977. The neutron star is a $\sim 680$ s X-ray pulsar (White et al. 1976), accreting from the dense wind of the optical companion. The wind's mass-loss rate of the optical component is one of the highest known in the galaxy: $\dot{M}_{\text {loss }} \sim 10^{-5} M_{\odot} \mathrm{yr}^{-1}$ (Kaper et al. 2006). Because the terminal velocity of the wind is very low $\left(w_{0} \sim 300-400 \mathrm{~km} \mathrm{~s}^{-1}\right.$, Kaper et al. 2006), the accretion rate is high enough to explain the observed luminosity of $L_{\mathrm{X}} \sim 10^{37} \mathrm{erg} \mathrm{s}^{-1}$. The distance to the source is estimated to be between $1.8 \pm 0.4 \mathrm{kpc}$ (Parkes et al. 1980) and $5.3 \mathrm{kpc}$ (Kaper et al. 1995), depending on the spectral classification of Wray 977. The latest estimate is $3 \mathrm{kpc}$ (Kaper et al. 2006). The orbit is highly eccentric with an eccentricity of $\sim 0.5$ and an orbital period of $\sim 41.5 \mathrm{~d}$ (Koh et al. 1997). The absence of X-ray eclipses despite the large radius $\left(R \sim 43 R_{\odot}\right)$ of Wray 977 (Parkes et al. 1980) constrains the inclination angle in the range $44-78^{\circ}$ with a best-fit value of $i \sim 66^{\circ}$ (Kaper et al. 2006; Leahy \& Kostka 2008). The source exhibits regular $\mathrm{X}$-ray flares about 1-2 d before the periastron passage (orbital phase $\sim 0.95$ ). There is also an indication of a second flare at orbital phase $\sim 0.5$ (Koh et al. 1997). Several hypotheses have been proposed to explain the observed orbital lightcurve, including a circumstellar disk (Koh et al. 1997) and a quasi-stable accretion stream (Leahy \& Kostka 2008). Similar to other wind accreting systems, the pulse period behavior of GX 301-2 on short time scales is described well by a random walk model (de Kool \& Anzer 1993). GX 301-2 exhibits a long-term pulse period evolution as well. The observed pulse period remained $\sim 700$ s until 1984 when it began to decrease during a rapid spin-up episode observed by BATSE (Koh et al. 1997; Bildsten et al. 1997). The spin-up trend reversed in 1993 (Pravdo \& Ghosh 2001) and ever since the pulse period has been increasing (La Barbera et al. 2005; Kreykenbohm et al. 2004).

The X-ray spectrum of the GX $301-2$ is rich in features. The lower energy range is subject to heavy and variable photoelectric absorption (White et al. 1976). As shown by Kreykenbohm et al. (2004) and La Barbera et al. (2005), a partial covering model with two absorption columns is required to describe the spectrum. There is a complex of iron lines at $\sim 6.4$ to $7.1 \mathrm{keV}$ (Watanabe et al. 2003). A high-energy cutoff at $\sim 20 \mathrm{keV}$, together with a deep and broad cyclotron resonance scattering feature (CRSF) at $\sim 30-45 \mathrm{keV}$, is present at higher energies (Makishima \& Mihara 1992; Orlandini et al. 2000; Kreykenbohm et al. 2004; La Barbera et al. 2005). The CRSF is highly variable with pulse phase, and it exhibits interesting correlations with the continuum parameters (Kreykenbohm et al. 2004).

The nature of accreting pulsars with long pulse periods is still poorly understood. Because of the low moment of inertia of the neutron star, the accelerating torque of the accreted matter can spin up a neutron star very efficiently. Braking torques are then required to explain the observed long pulse periods. It is commonly assumed that the observed pulse period is determined by the equality of torques affecting the neutron star or relaxes to the value determined by this equality. Braking torques 
Table 1. Pulse period values obtained with archival INTEGRAL data.

\begin{tabular}{llll}
\hline \hline $\mathrm{MJD}_{\text {obs }}$ & Period, s. & $\mathrm{MJD}_{\text {obs }}$ & Period, s. \\
\hline 52833.5 & $681.33 \pm 0.04$ & 53538.0 & $684.59 \pm 0.12$ \\
53088.0 & $683.42 \pm 0.07$ & 53541.6 & $684.4 \pm 0.04$ \\
53525.9 & $683.9 \pm 0.1$ & 53545.6 & $684.27 \pm 0.04$ \\
53528.5 & $684.19 \pm 0.16$ & 53549.4 & $684.15 \pm 0.05$ \\
53529.7 & $684.15 \pm 0.44$ & 54111.9 & $684.62 \pm 0.06$ \\
53535.0 & $684.73 \pm 0.05$ & 54277.6 & $685.15 \pm 0.07$ \\
\hline
\end{tabular}

Notes. An updated ephemeris was used to correct the lightcurve for binary motion. The error is estimated as $10 \%$ of the width of the peak in the periodogram.

Table 2. Pointed observations of GX 301-2 by INTEGRAL, with an updated ephemeris to calculate the orbital phase.

\begin{tabular}{lllll}
\hline \hline $\begin{array}{l}\text { INTEGRAL } \\
\text { science window }\end{array}$ & $\begin{array}{l}\text { MJD of } \\
\text { observation }\end{array}$ & $\begin{array}{l}\text { Orbital } \\
\text { phase }\end{array}$ & $\begin{array}{l}\text { Exposure, } \\
\text { ksec }\end{array}$ & $\begin{array}{l}\text { Rate, } \\
\text { cts s }^{-1}\end{array}$ \\
\hline $05180027-66$ & $54110.5-12.2$ & $0.96-1.02$ & 91.66 & 98 \\
$05730048-60$ & $54276.3-76.9$ & $0.95-0.97$ & 31.98 & 163 \\
$05740012-40$ & $54277.6-78.9$ & $0.99-1.02$ & 69.38 & 76 \\
\hline
\end{tabular}

Notes. ${ }^{(*)}$ In $20-60 \mathrm{keV}$ energy range using the ISGRI lightcurve.

are generally associated with the coupling of the neutron star's magnetic field with the surrounding plasma. The drag force depends on the relative linear speed of field lines at certain effective radius, which in turn depends on the magnetic field strength. The efficiency of braking decreases for slowly rotating and weakly magnetized neutron stars so a strong field (up to $10^{15} \mathrm{G}$, Shakura 1975 ) is required to spin down a slowly rotating accreting X-ray pulsar even further. This results in an apparent contradiction with field estimates obtained from the CRSF centroid energy, which is $B \sim\left(E_{\text {cyc }} \mathrm{keV} / 11.57\right) \times 10^{12} \mathrm{G} \sim 4 \times 10^{12} \mathrm{G}$ in the case of GX 301-2 and in the same order of magnitude as for other sources.

We suggest that this contradiction may be resolved if the line-forming region resides in an accretion column of significant height (Basko \& Sunyaev 1976), comparable to the neutron star radius. We investigate this hypothesis using INTEGRAL and $B A T S E$ observations to study the spectral and timing properties of GX 301-2.

\section{Observations and data selection}

The International Gamma-Ray Astronomy Laboratory (INTEGRAL) launched in October 2002 by the European Space Agency (ESA) is equipped with 3 co-aligned coded mask instruments: IBIS (Imager onboard the INTEGRAL Satellite, Ubertini et al. 2003), JEM-X (Joint European X-ray Monitor, Lund et al. 2003), and SPI (Spectrometer on INTEGRAL, Vedrenne et al. 2003). Because of limited SPI sensitivity for variable sources, we rely on data from IBIS (the ISGRI layer) and $J E M-X$ in this paper. Among the INTEGRAL instruments, $I B I S$ has the largest field of view and, therefore, the highest probability of observing the source. We used a total of 554 available public pointings with GX 301-2 within the IBIS half-coded field of view for the pulse period determination (i.e. for Table 1). These data include a long observation that covers $\sim 60 \%$ of the orbital cycle and is long enough to allow binary ephemeris estimation (283 pointings in INTEGRAL revolutions 322-330). Three dedicated observations (see Table 2) were also performed during the pre-periastron flare and were used to study the spectrum of the source.

We also used results provided by the ASM/RXTE teams at $M I T$ and at the RXTE SOF and GOF at NASA's GSFC and CGRO BATSE pulsar DISCLA histories data by Bildsten et al. (1997) to study the long-term evolution of the spin period.

\section{Observational results}

\subsection{Timing analysis}

To derive the intrinsic pulse period of the source, the lightcurve must be corrected for Doppler delays due to the orbital motion of the source and the satellite. Phase connection or pulse time arrival analysis is a precise timing technique, based on measuring arrival times of individual pulses or groups of pulses (Staubert et al. 2009). It allows to determine the Doppler delays and therefore the orbital parameters of the system. A fixed phase of the pulsating flux from a pulsar is observed at times (Nagase et al. 1982):

$$
\begin{aligned}
T_{n}= & T_{0}+P_{0} n+\frac{1}{2} \dot{P} P_{0} n^{2}+\frac{1}{6} \ddot{P} P_{0}{ }^{2} n^{3} \ldots \\
& +a \sin (i) F_{n}\left(e, \omega, T_{\mathrm{PA}}, \theta\right)
\end{aligned}
$$

referred to as Time Of Arrival (TOA), where $P_{0}, \dot{P}$, and $\ddot{P}$ are the intrinsic pulse period and its time derivatives at the initial epoch $T_{0}$. The last term represents the Doppler delays due to the orbital motion as a function of the Kepler parameters for an eccentric orbit: the projected semi-major axis $a \sin i$ in light seconds ( $i$ is the orbit inclination), the eccentricity $e$, the longitude of the periastron $\omega$, time of periastron passage $T_{\mathrm{PA}}$, and the mean anomaly $\theta=2 \pi\left(T-T_{\mathrm{PA}}\right) / P_{\text {orb }}$. To obtain a solution for the unknown pulse and orbital parameters, a number of measurements of $T_{n}$ (for known $n$ ) must be obtained. Usually only $T_{n \text {,obs }}$ is measured, while $n$ must be found during the fitting procedure to obtain a self-consistent solution. The orbital period may be estimated either directly as one of the free parameters or by comparing periastron passage times of subsequent cycles (i.e. similarly to the pulse period). The latter method is more precise (see e.g. Staubert et al. 2009).

Using archival ISGRI observations and the standard OSA 6.1 software provided by $I S D C^{1}$, we constructed lightcurves with $20 \mathrm{~s}$ time bins in the energy range $20-40 \mathrm{keV}$ and determined the pulse arrival times (each pulse profile obtained by folding $\sim 20$ individual pulses) for data from revolutions 322-330 using a technique similar to the one by Koh et al. (1997). This is the only INTEGRAL observation to cover a significant fraction of the orbital cycle, and it allows estimation of binary parameters. We then used Eq. (1) to determine $P, \dot{P}$ and $T_{\mathrm{PA}}$. The other orbital parameters were fixed to values reported by Koh et al. (1997). Our best-fit values are $P_{\text {pulse }}=684.1618(3) \mathrm{s}, \dot{P}_{\text {pulse }}=4.25(22) \times 10^{-8} \mathrm{~s} \mathrm{~s}^{-1}$ at the epoch 53523.8, and $T_{\mathrm{PA}}=53531.63(1) \mathrm{MJD}$. All uncertainties are at $1 \sigma$ confidence level unless otherwise stated. Pulse delays from the orbital motion and residuals of the best-fit are plotted in Fig. 1. Comparing our $T_{\mathrm{PA}}$ value with the historical values reported by White \& Swank (1984), Sato et al. (1986) and Koh et al. (1997) allows estimation of the orbital period. Inclusion of our measurement requires introducing of a secular change to the orbital period. The quality of the fit improves significantly ${ }^{2}$. The residuals to fit both with and without inclusion

\footnotetext{
1 http://isdc.unige.ch

2 The $\chi^{2}$ drops from 52 to 0.52 with an F-test significance of $\sim 98 \%$.
} 


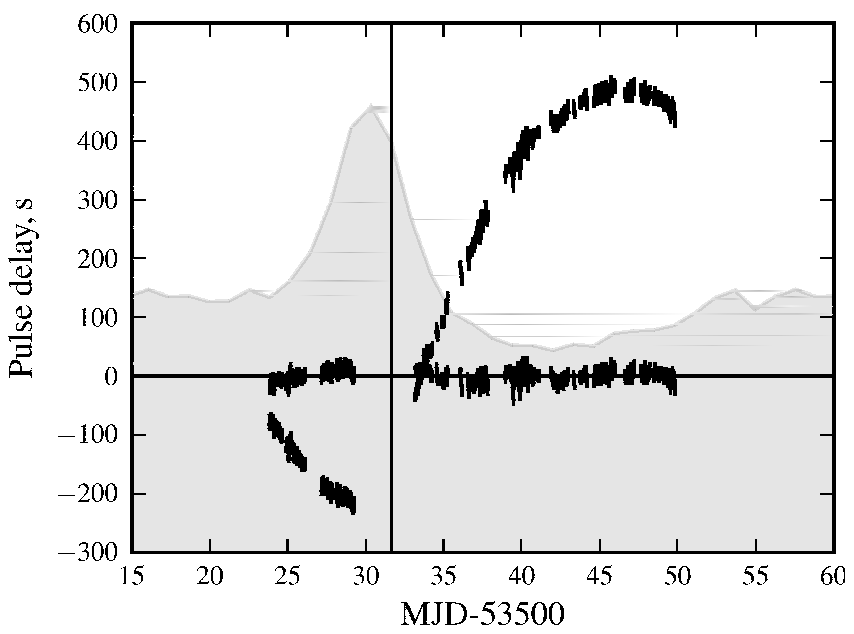

Fig. 1. Time delays of pulse arrival times induced by the orbital motion. Changes due to the intrinsic variation of the spin period are subtracted. best-fit residuals are also shown. The best-fit periastron passage time is marked with a vertical line. The folded RXTE ASM orbital profile with the pre-periastron flare is plotted in gray.

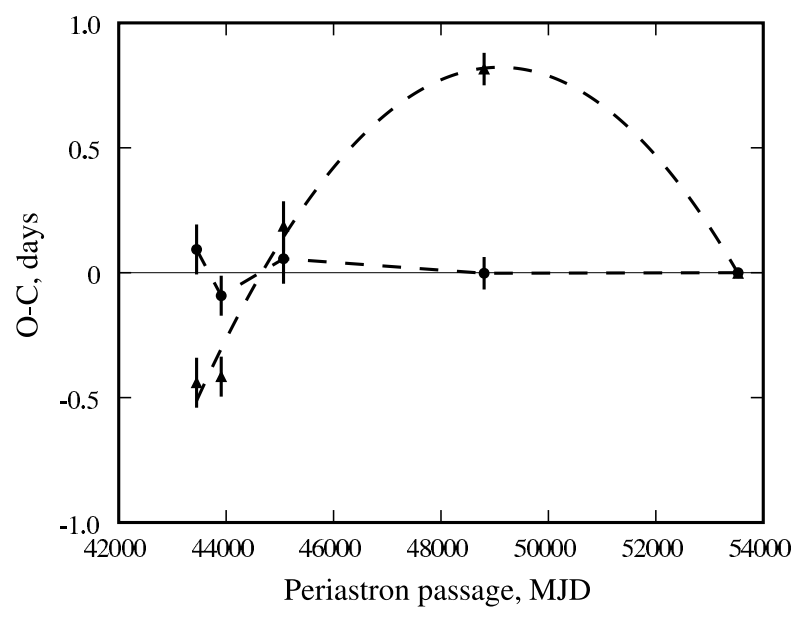

Fig. 2. Residuals to fit over periastron passage times for orbital period with (circles) and without (triangles) inclusion of the orbital period derivative.

of a secular change are plotted in Fig. 2. Our best fit values are $P_{\text {orb }}=41.506 \pm 0.003 \mathrm{~d}$ and $\dot{P}_{\text {orb }}=(-3.7 \pm 0.5) \times 10^{-6} \mathrm{~s} \mathrm{~s}^{-1}$ at the reference time reported by Sato et al. (1986): $T_{\mathrm{PA}, 0}=$ $43906.06 \pm 0.11$. This estimate is consistent with the direct measurements of the orbital period both by Sato et al. (1986) and by Koh et al. (1997).

It should be emphasized that the commonly used value of $P_{\text {orb }}=41.498 \mathrm{~d}$ by Koh et al. (1997) was obtained by comparison of the $T_{\mathrm{PA}}$ values as well (the authors compared their value to that by Sato et al. (1986) under the assumption of a constant orbital period). On the other hand, all published measurements including ours are consistently described when an orbital period derivative is included. For the time of the INTEGRAL observation, the predicted orbital period is $\sim 41.472 \mathrm{~d}$. The periastron passage time measured with the orbital period value fixed to this prediction does not change significantly: $T_{\mathrm{PA}}=53531.65 \pm 0.01 \mathrm{MJD}$.

Because the pre-periastron flare in the orbital lightcurve of the source is associated with the periastron passage time, an additional check can be made using the long-term lightcurve of

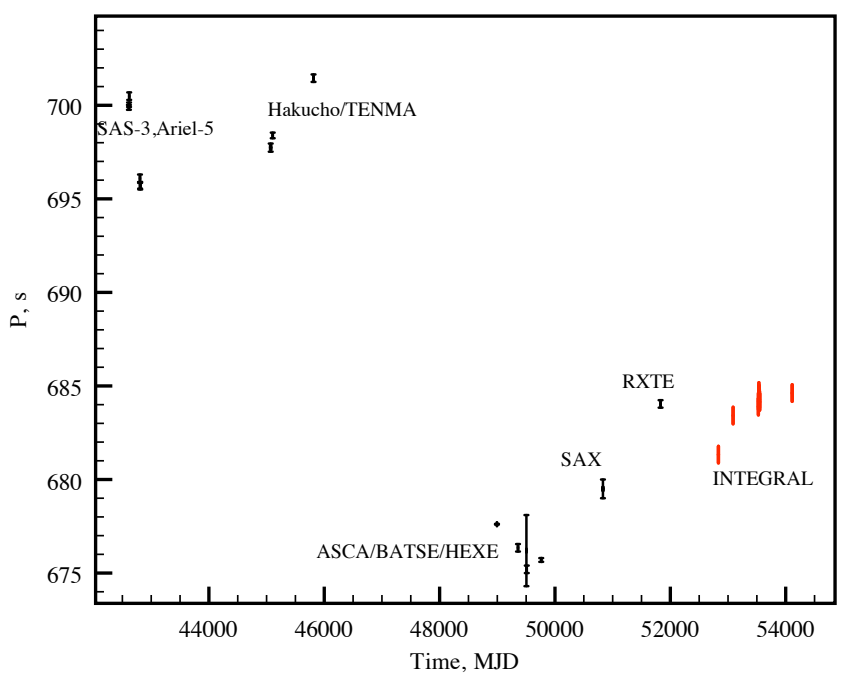

Fig. 3. Long-term pulse period evolution of GX 301-2.

the source. We split a 10-year long RXTE ASM barycentered daily lightcurve of GX 301-2 (all bands combined) into parts of $\sim 5$ orbital cycles in length and folded each part with the orbital period $P_{\text {orb }}=41.498 \mathrm{~d}$ to obtain a series of orbital profiles. The relative phase shifts and the associated orbital periods were then determined in the same way as for the pulse period. The bestfit value for a constant period is $P_{\text {orb }}=41.482 \pm 0.001 \mathrm{~d}$. The mean value of the orbital period, calculated using $P_{\text {orb }}$ and $\dot{P}_{\text {orb }}$ obtained above, is consistent with the observed value at the time of the $A S M$ observations, although an orbital period derivative is not formally required by the $A S M$ data alone.

A set of pulse period measurements with epoch folding was performed with the updated ephemeris. We grouped all available INTEGRAL data by the observation time by the " $k$-means" clustering algorithm (MacQueen 1967). The number of groups was chosen such that the mean number of X-ray pulses within one group was $\sim 50$. We searched for pulsations in each of the groups with epoch folding (Larsson 1996). A few groups where no pulsations were found because of insufficient statistics were rejected afterwards. The results are listed in Table 1 and plotted in Fig. 3 together with historical values for clarity. On average, GX 301-2 continues to spindown after the first INTEGRAL observation of the source.

\subsection{Spectral analysis}

The observations listed in Table 2 were used to obtain the broadband spectrum of the source. Since all three observations were made at almost the same orbital phase, we combined all data to have better statistics. We used the standard OSA 6.1 pipeline for spectral extraction. A systematic error of $1 \%$ for all ISGRI and of $2 \%$ for all $J E M-X$ spectra was assigned as suggested in the OSA documentation.

Spectra of X-ray pulsars are usually described with phenomenological multi-component models. The continuum of GX 301-2 was been modeled with a cut-off power law modified at low energy by photoelectric absorption. An iron emission $K_{\alpha}$ line was been also observed. In fact, there is a complex of iron lines at $\sim 6.4 \sim 7.1 \mathrm{keV}$ (Watanabe et al. 2003; La Barbera et al. 2005) in the spectrum of GX 301-2. These are not resolved with $J E M-X$. We therefore used a simple Gaussian-shaped profile with larger width to formally describe this feature. 
The photoelectric absorption of the source's spectrum is strongly variable, and at least two absorption columns are identified. Part of the X-ray emission is thought to be strongly absorbed close to the neutron star, while all emission from this region is also subject to absorption in the overall stellar wind of the optical companion. A model describing this physical situation is the absorbed partial covering model (Kreykenbohm et al. 2004; La Barbera et al. 2005).

From a more physical point of view, the spectrum of an accreting pulsar is believed to be mainly the result of a Comptonization processes of thermal photons in the accretion column and in the neutron star atmosphere. The emerging spectrum depends on the optical depth and generally has a power-law shape, with a cut-off at an energy corresponding to the temperature of the Comptonizing medium ( $\sim 3 k T_{\mathrm{e}}$, Sunyaev \& Titarchuk 1980). Phenomenological models aim at describing this shape regardless of the optical depth. For GX 301-2 two models have been used in literature. La Barbera et al. (2005) adopted a modified "high energy" cut-off, while Kreykenbohm et al. (2004) used a so-called Fermi-Dirac cut-off. As discussed in Doroshenko et al. (2008), both models describe the INTEGRAL data well with parameters close to the published ones. It is somewhat difficult, however, to interpret these results from a physical point of view. We therefore focus here on a different description.

One of the first physical models to describe Comptonization spectra was proposed by Sunyaev \& Titarchuk (1980). Compton scattering in strong magnetic field is a more complicated problem (Lyubarsky 1986; Meszaros \& Nagel 1985), but for the saturated case $\left(\tau_{\mathrm{e}} \gg 1\right)$ a blackbody-like spectrum is formed in both cases (Lyubarsky 1986). The Sunyaev \& Titarchuk (1980) model is included in the standard $X S P E C$ distribution as COMPST. Free parameters include the electron temperature of the medium $T_{\mathrm{e}}$, optical depth $\tau_{\mathrm{e}}$, and normalization $A_{\mathrm{st}}$. We used this model because it contains the least number of free parameters and produces identical results to more complex models for GX 301-2. The pulse-phase averaged spectrum was extracted and fitted with the partially absorbed COMPST model. The fit results are listed in Table 3. Since the optical depth of the Comptonizing medium is very high, we verified that a simple black body model provides an equally good description of the data. The unabsorbed source flux in the same energy range is $\sim 1.8 \times 10^{-8} \mathrm{erg} \mathrm{cm}^{-2} \mathrm{~s}^{-1}$ in both models.

A CRSF was necessary in the fit. This was included assuming a Gaussian-shaped profile. With the inclusion of the line the $\chi_{\text {red }}^{2}$ dropped from $\sim 3.8$ (depending on the model) to values around 1.2 (see Table 3 ).

\section{Discussion}

\subsection{Orbital period evolution}

Our estimate of the rate of orbital period decay $\dot{P}_{\text {orb }} / P_{\text {orb }} \simeq$ $-3.25 \times 10^{-5} \mathrm{yr}^{-1}$ exceeds that of other known sources at least by one order of magnitude. Previous detections include Cen $\mathrm{X}-3$ $\left(\dot{P}_{\text {orb }} / P_{\text {orb }}=-1.738 \times 10^{-6} \mathrm{yr}^{-1}\right.$ (Bagot 1996 , and references therein $),$ SMC X-1 $\left(\dot{P}_{\text {orb }} / P_{\text {orb }}=-3.36 \times 10^{-6} \mathrm{yr}^{-1}\right)$ and Her X-1 with $\dot{P}_{\text {orb }} / P_{\text {orb }}=-1.0 \times 10^{-8} \mathrm{yr}^{-1}$ (Staubert et al. 2009). GX 301-2 is very different from all these systems. It is younger and has a highly eccentric orbit, while other systems have almost circular orbits. Both real and apparent changes in the orbital period are expected to be greater for an eccentric orbit.

We measured the rate of decay of the orbit by comparing the times of several periastron-passages. Those are determined by fitting the observed pulse delays as a function of orbital phase,
Table 3. Fit results for phase averaged spectra. Uncertainties are expressed at $90 \%$ confidence level.

\begin{tabular}{lll}
\hline \hline Parameter & $\begin{array}{l}\text { Absorbed } \\
\text { blackbody }\end{array}$ & $\begin{array}{l}\text { Absorbed } \\
\text { COMPST }\end{array}$ \\
\hline$N_{\mathrm{H}, 1}\left[10^{22}\right.$ atoms $\left./ \mathrm{cm}^{2}\right]$ & $\leq 4$ & $\leq 4$ \\
$N_{\mathrm{H}, 2}\left[10^{22}\right.$ atoms $\left./ \mathrm{cm}^{2}\right]$ & $178.3_{-6.7}^{+6.9}$ & $175.6_{-9.9}^{+10.4}$ \\
$c_{\mathrm{F}}$ & $0.798_{-0.008}^{+0.008}$ & $0.78_{-0.01}^{+0.01}$ \\
$E_{\text {gabs }}[\mathrm{keV}]$ & $45.8_{-1.6}^{+1.7}$ & $45.9_{-1.6}^{+1.8}$ \\
$\sigma_{\text {gabs }}[\mathrm{keV}]$ & $15.0_{-1.7}^{+1.8}$ & $15.1_{-1.8}^{+2.0}$ \\
$d_{\text {gabs }}$ & $57.45_{-17.89}^{+23.66}$ & $57.93_{-18.19}^{+25.93}$ \\
$A_{\mathrm{bb} / \mathrm{st}}$ & $0.31_{-0.06}^{+0.06}$ & $0.35_{-0.05}^{+0.09}$ \\
$T_{\mathrm{e}}[\mathrm{keV}]$ & $5.1_{-0.2}^{+0.2}$ & $5.1_{-0.2}^{+0.3}$ \\
$\tau_{\mathrm{e}}$ & - & $42.3_{-3.9}^{+4.8}$ \\
$E_{\mathrm{Fe}}[\mathrm{keV}]$ & $6.32_{-0.02}^{+0.02}$ & $6.32_{-0.02}^{+0.02}$ \\
$\sigma_{\mathrm{Fe}}[\mathrm{keV}]$ & $0.36_{-0.05}^{+0.04}$ & $0.38_{-0.04}^{+0.04}$ \\
$\chi^{2} /$ d.o.f. & $1.18 / 149$ & $1.17 / 148$ \\
\hline
\end{tabular}

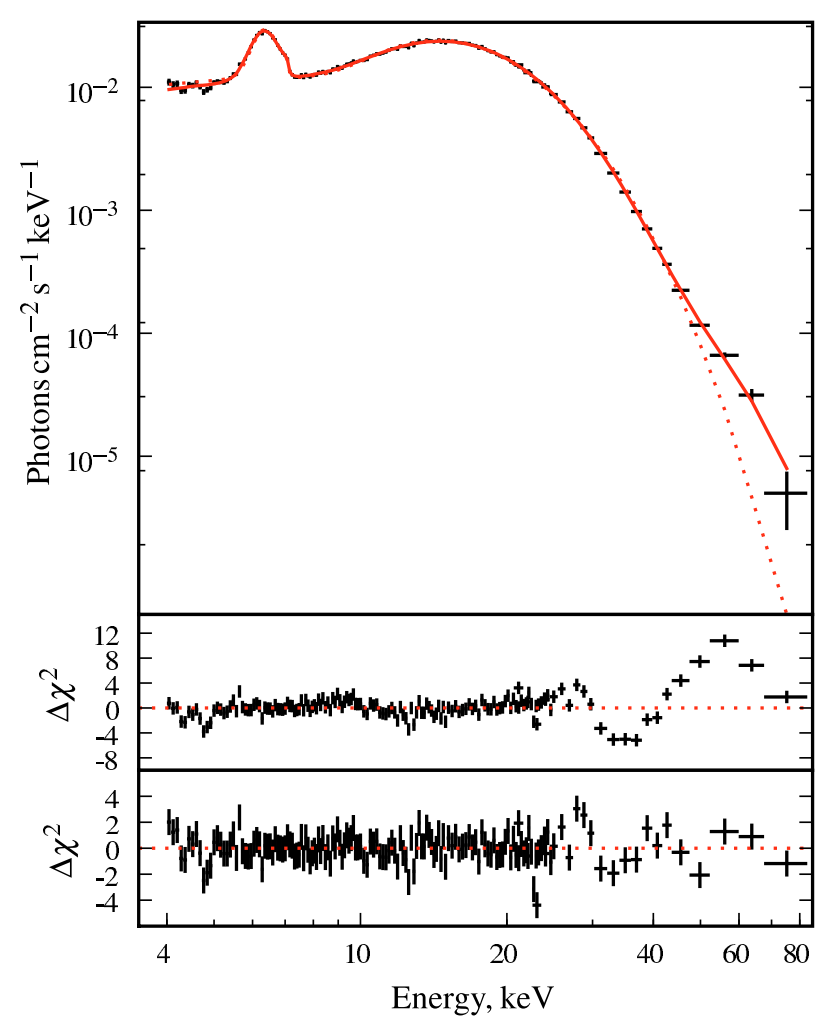

Fig. 4. Unfolded spectrum and residuals for the COMPST model without (dotted line and second panel) and with the inclusion (solid line and bottom panel) of a CRSF at $\sim 46 \mathrm{keV}$, using ISGRI $(20-80 \mathrm{keV})$ and $J E M-X(4-20 \mathrm{keV})$ data.

and they may in principle be correlated with other model parameters, particularly with the longitude of periastron due to apsidal motion. The span of our data used for the pulse time arrival analysis does not allow both $T_{\mathrm{PA}}$ and $\omega$ to be reliably constrained simultaneously. All published estimates of $\omega$ are also consistent with each other within uncertainties, but still we cannot rule out that apsidal motion contributes to the observed apparent change in the orbital period.

The eccentric orbit and very strong mass transfer in the system (the mass loss by the optical component is $\dot{M}_{\text {loss }} \sim$ $10^{-5} M_{\odot} \mathrm{yr}^{-1}$, Kaper et al. 2006) suggest that some intrinsic changes in the orbital period are also expected. 
The optical companion is much heavier than the neutron star and contributes almost nothing to the orbital angular momentum of the system. Direct mass loss via the stellar wind by the optical companion therefore does not lead to significant loss of the angular momentum. The optical star becomes less massive, leading to a longer orbital period (Hilditch 2001), which is the opposite of what is observed, although the rate of such change is very low.

To explain the decrease in the orbital period, one has to assume that the material carrying the angular momentum away must come from the vicinity of the neutron star, since it is the neutron star's orbital motion that represents the bulk of the angular momentum in the system. We can see two mechanisms that could be responsible for the loss of angular momentum. First, material of the stellar wind that is streaming by the neutron star feels the gravitational pull of the moving neutron star. Only a fraction of this material is eventually accreted onto the neutron star, and the larger part is leaving the binary system and carrying some angular momentum away, since the interaction with the neutron star changed its trajectory. Second, before the matter is accreted onto the neutron star, it interacts with the neutron star's magnetosphere (leading to spin-up and spin-down of the neutron star, as will be discussed below). However, the interaction with the magnetosphere may also lead to a magnetically driven outflow of material (Illarionov \& Kompaneets 1990; Lovelace et al. 1999; Klochkov et al. 2009), again carrying angular momentum away. In addition, tidal coupling of the rotational frequency of the optical star with the orbital frequency could play some role, although estimates by Leahy \& Kostka (2008) and Hilditch (2001) suggest that, despite the high eccentricity, this is probably not very efficient. The details of the mass transfer and angular momentum loss in this system are not understood well, and more observations are required to secure the rate of change in the orbital period.

\subsection{Torque balance and magnetic moment of the neutron star}

The evolution of the spin frequency of the neutron star gives insight into the interaction of the accretion flow with the neutron star. The rotational dynamics is determined by the equation

$I \frac{\mathrm{d} \omega}{\mathrm{d} t}=K_{+}+K_{-}$

where $K_{+}$and $K_{-}$are the acceleration and deceleration torques, where $\omega$ is the pulse frequency, and $I$ the momentum of inertia of the neutron star.

Two rapid spin-up episodes observed by Koh et al. (1997) indicate that a long lived accretion disk may sometimes form in GX 301-2. Both episodes are characterized by an increased source flux, which implies an increased accretion rate. The infrequent occurrence of such episodes argues against the hypothesis that they are triggered by tidal overflows at periastron (see Layton et al. 1998) and suggests that mass loss episodes of Wray 977 may be responsible for them (Koh et al. 1997). As concluded by Koh et al. (1997), the pulse period decrease in 1984-1992 can be attributed entirely to similar spin-up episodes, while most of the time the neutron star accretes from the wind, and no net change of the pulse period is observed. It is therefore important to understand the torque balance in this case. This is why we focus on wind-accretion models.

In the case of quasi-spherical accretion from a stellar wind the accelerating torque can be expressed as (Davies et al. 1979)

$K_{+}=\dot{M} k_{w} R_{A}^{2} \Omega_{\text {orb }}$ where $\Omega_{\text {orb }}$ is the orbital frequency, $\dot{M}$ the accretion rate, $k_{w}$ dimensionless coefficient reflecting the efficiency of the angular momentum transfer, and $R_{A}$ the accretion radius given by

$R_{A}=\frac{2 G M}{v_{\mathrm{rel}}^{2}}$,

where $B$ is the field strength, $R, M$ are the neutron star radius and mass, and $v_{\text {rel }}$ is the relative speed of the wind and the neutron star. Hydrodynamical simulations (Taam \& Fryxell 1989; Fryxell \& Taam 1988; Ruffert et al. 1992; Ruffert 1997) show that, due to the asymmetry of the accretion flow caused by the orbital motion of the neutron star and due to fluctuations in the speed and density of the local wind, short lived accretion disks may form. The disk direction alternates between prograde and retrograde depending on the local physical conditions. This causes the neutron star to alternate between short spin-up (prograde disk) and spin-down (retrograde disk) episodes, so a significant fraction of the angular momentum carried by the wind is cancelled out. To account for this decrease in the efficiency of angular momentum transfer, $k_{w}$ is introduced in Eq. (3). Its value is a controversial topic, but simulations predict that it is rather small (absolute value less than $\sim 1.2$ according to Ho 1989).

There are several models for the deceleration torque. According to Davies et al. (1979) and Bisnovatyi-Kogan (1991), an asymmetric magnetosphere of the accreting pulsar produces turbulent viscosity in the nearby wind, which brakes the neutron star:

$K_{-}=-\dot{M} \frac{\omega^{2} R_{\mathrm{m}}{ }^{7 / 2}}{4 \sqrt{2 G M}}$

where $R_{\mathrm{m}}$ is the magnetospheric radius given by

$R_{\mathrm{m}}=\left(\frac{B^{2} R^{6}}{2 \dot{M} \sqrt{2 G M}}\right)^{2 / 7}$.

Instead, Illarionov \& Kompaneets (1990) explain the spin down as a result of the efficient angular momentum transfer from the rotating magnetosphere of the accreting star to an outflowing stream of magnetized matter. This outflow is said to be a result of the heating of the accreting matter by hard X-ray emission from the pulsar through Compton scattering. The outflow is focused within a certain solid angle owing the anisotropy of the pulsar emission. It has a lower density than the surrounding accreting matter due to the higher temperature, thus it is driven out by the buoyancy force. Angular momentum gain from the accreting gas is balanced by angular momentum loss via the outflow, enabling a spin-down of the neutron star under certain circumstances. In this model,

$K_{-}=-\dot{M} k \frac{\xi}{2 \pi} R_{\mathrm{m}}^{2} \omega$,

where $\xi \sim 1$ is the solid angle of the stream outflow. The dimensionless coefficient $k \sim 2 / 3$ accounts for the orientation of the outflow with respect to the magnetic field. It is worth noting, that the equilibrium period obtained from the comparison of this torque with the accelerating torque has the same value (aside from the numerical coefficients) as second estimate by Bisnovatyi-Kogan (1991). The braking torque there arises from the turbulent viscosity in the same way as in Eq. (5), but it is assumed, that the magnetosphere and accreting matter corotate, and the velocity for the velocity dependent viscosity coefficient is taken as the sound speed for the corresponding temperature at the magnetosphere boundary (and not as relative velocity 
of the wind and the magnetosphere). The angular momentum is then carried away with the turbulent motions. As noted by Bisnovatyi-Kogan (1991), the situation for real pulsars is probably somewhere in between these two estimates. The braking torques in both models may also include undetermined coefficients $\sim 1$, which, can however, be incorporated into the accelerating torque term.

The torque balance, hence the rotational frequency derivative, depends on $\dot{M}$, so one has to investigate this dependence to study the rotational dynamics. Since the longest continuous pulse frequency monitoring campaign $(\sim 10 \mathrm{yr})$ for this source was carried out with BATSE (Bildsten et al. 1997), we used the data products available at the CGRO mission web-page ${ }^{3}$. The pulse frequency and pulse frequency derivative histories are provided for the entire BATSE lifetime. Both were determined for a set of $\sim 4 \mathrm{~d}$ intervals using the phase connection technique assuming the ephemeris by Koh et al. (1997) for binary-motion corrections (see Bildsten et al. 1997; Koh et al. 1997 for details). The corresponding BATSE pulsed flux in the $20-50 \mathrm{keV}$ energy range, averaged over the interval is also provided.

Contrary to the report by İnam \& Baykal (2000), a correlation between the angular frequency derivative $(\dot{\omega}=2 \pi \dot{v})$ and the flux (see Fig. 5) was found (Pearson correlation coefficient 0.96, null hypotheses probability $8 \times 10^{-6}$ ).

The discrepancy between our findings and the ones reported in İnam \& Baykal (2000) lie in their method of estimating frequency derivatives. With the BATSE data set that we used, Inam $\&$ Baykal (2000) estimate pulse frequency derivatives by grouping the provided frequency values in intervals of $\sim 30 \mathrm{~d}$ and averaging between the left and right frequency derivatives calculated using these values for each interval. This approach is incorrect because it assumes that the frequency values provided by Koh et al. (1997) alone characterize the average pulse frequency during the corresponding observation time, while the average pulse frequency also depends on the frequency derivative included in the fit and on the observation length. For this approach to work it is required to remove the frequency derivative in the fit for the pulse arrival times in the raw BATSE data, which was not done by İnam \& Baykal (2000). There is also a second point to question in their analysis. To obtain values of the first derivative, İnam \& Baykal (2000) use frequency values on intervals of $\sim 30 \mathrm{~d}$, comparable to the orbital period of the system. Both the pulse frequency and the flux are known to change on much shorter time scales in GX 301-2. Averaging on such a long interval smoothes out most of the flux and pulse frequency variations, making it difficult to find the correlation between the two quantities.

On the other hand, we used $\dot{\omega}$ and flux values directly measured for each observation with phase connection. The points in Fig. 5 were obtained by averaging provided frequency derivative values of points with flux in a given range. The standard error was used as an uncertainty estimate. We excluded both spin-up episodes observed by Koh et al. (1997) (i.e. MJD 48440-48463 and MJD 49230-49245) and intervals where pulsations were not detected reliably (see Koh et al. 1997) from the further analysis.

To investigate the accretion models and compare them to the data, we need to express the accretion rate as a function of the count rate, not a trivial task. The conversion depends on the distance, radiative efficiency of accretion, and beaming factor. We assumed that the mean source flux derived from the spectra

\footnotetext{
$3 \mathrm{ftp}: / /$ heasarc.gsfc.nasa.gov/compton/data/batse/ pulsar/histories/DISCLA_histories/gx301m2_psr_hist. fits
}

obtained with the INTEGRAL pointed observations corresponds to the mean BATSE count-rate at the same orbital phase. Then we assumed a conversion factor of $10^{37} \mathrm{erg} \mathrm{s}^{-1} \simeq 10^{17} \mathrm{~g} \mathrm{~s}^{-1}$ which corresponds to the radiative efficiency of accretion $\sim 10 \%$, $\left.L_{\mathrm{x}} \sim 0.1 \dot{M} \mathrm{c}^{2}\right)$ to estimate the accretion rate. The distance to the source is uncertain so the derived value should account for the spread of the estimates (1.4-5.3 kpc). Apparently, $K_{+}$, hence the torque balance, depends significantly on the efficiency of angular momentum transfer $k_{w}$ and on the relative velocity of the neutron star and the wind.

The orbit of GX 301-2 is eccentric, so the orbital speed of the neutron star changes significantly along the orbit. The wind, on the other hand, is also accelerated from the sound speed at the surface of Wray $977\left(\sim 10 \mathrm{~km} \mathrm{~s}^{-1}\right)$ to a terminal velocity of $300-400 \mathrm{~km} \mathrm{~s}^{-1}$ at infinity (Castor et al. 1975):

$v_{w}(r)=v_{0}+\left(v_{\infty}-v_{0}\right)\left(1-R_{*} / r\right)^{\beta}$

where $v_{0}$ is the velocity at the surface of the star close to the sound speed, $v_{\infty}$ the terminal wind speed, and $\beta \sim 1$ for O-type stars. Radial and tangential components of the neutron star as function of orbital phase $\theta$ are

$v_{r}=\sqrt{\frac{\mu}{p}} e \sin \theta, \quad v_{t}=\sqrt{\frac{\mu}{p}}(1+e \cos \theta)$

where $\mu=G\left(M_{\mathrm{opt}}+M_{\mathrm{NS}}\right), p=a\left(1-e^{2}\right), e$ is the eccentricity, and $a \sim 1.2 \times 10^{13} \mathrm{~cm}$ is the semi-major axis for $i=66^{\circ}$. It turns out that, while both the orbital speed of the neutron star and the wind speed are strong functions of the orbital phase, the relative velocity varies only by a factor of 2 (see Fig. 6). Each flux bin in Fig. 5 contains measurements performed at different orbital phases. We then calculated an average relative speed for each flux bin to properly estimate $R_{A}$. It turns out, however, that the relative speed varies only within $5 \%$ and consequently $R_{A}$ does not changes significantly (see Fig. 5).

The absolute value of $k_{w}$ cannot be arbitrarily small, otherwise the source will not be able to spin up, while this is clearly the case when the flux exceeds a certain value. The value of $k_{w}$ can, therefore, be estimated using the observed frequency derivative over accretion rate dependence. We only measure flux, so the accretion rate and $k_{w}$ are parametrized by the assumed distance. For each distance in the range of published estimates from 1.4 to $5.3 \mathrm{kpc}$, we calculated the accretion rate and estimated $k_{w}$ and $B$ as free parameters of models defined by Eqs. (3)-(7) where we assumed $M=1.4 M_{\odot}, R=10^{6} \mathrm{~cm}, I=1.4 \times 10^{45} \mathrm{~g} \mathrm{~cm}^{2}$, $v_{\infty}=300 \mathrm{~km} \mathrm{~s}^{-1}, k=2 / 3$, and $\xi=0.87$. The estimated values are presented in Fig. 7. The $k_{w}$ range is in line with estimates obtained by Ho (1989) and with later claims that the average amount of angular momentum transferred from the wind to the neutron star is relatively small (Ruffert et al. 1992; Ruffert 1997). It is likely that the mechanisms to generate the braking torques assumed in the models may act simultaneously, so we attempted to find the magnetic field required in this case by including both torques. The required field strength, however, is not significantly reduced and still exceeds $10^{14} \mathrm{G}$ (see Fig. 7).

It is important to emphasize that the frequency derivative and therefore torque affecting the neutron star are consistent with zero for the average source flux. This means that, for the average conditions during the observations, the period is close to a so-called equilibrium period (i.e. when the torques are balanced). This is also in line with the long-term pulse period evolution (see Fig. 3). The knowledge of the equilibrium period allows estimation of the magnetic field for the average luminosity even without knowing the exact dependency of torque with 


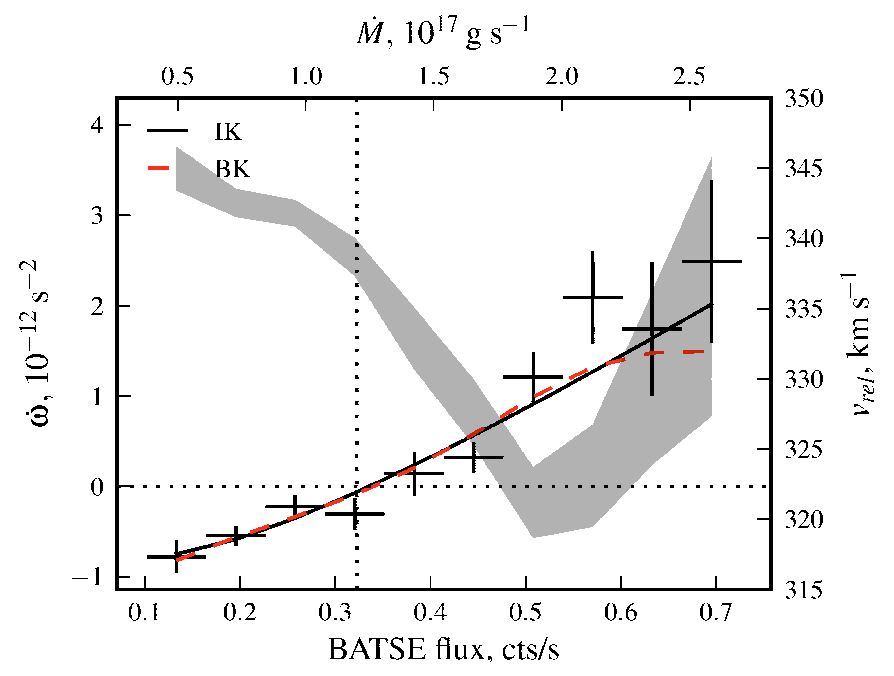

Fig. 5. Angular frequency derivative correlation with flux. Flux is BATSE-pulsed flux in the 20-40 keV energy range. BATSE DISCLA data provided by Bildsten et al. (1997) on the GGRO mission page are used to obtain the plot. Example of fitting Illarionov \& Kompaneets (1990) (solid black) and Davidson \& Ostriker (1973); Davies et al. (1979); Bisnovatyi-Kogan (1991) (dashed red) models for an assumed distance of $3 \mathrm{kpc}$ is plotted. Top axis shows the estimated accretion rate for this distance. The vertical line indicates the mean flux during the observation. Shaded area represents average relative velocity of the neutron star and wind for a given flux bin (right scale).

luminosity. For example, for the Davidson \& Ostriker (1973), Davies et al. (1979), Bisnovatyi-Kogan (1991) model in the case of torque equivalence, the field strength may be expressed as

$$
\begin{aligned}
B \approx & 3 \times 10^{14} \mathrm{G}\left(\frac{k_{w}}{0.25}\right)^{1 / 2}\left(\frac{\dot{M}_{\mathrm{eq}}}{10^{17} \mathrm{~g} / \mathrm{s}}\right)^{1 / 2}\left(\frac{v_{\mathrm{rel}}}{400 \mathrm{~km} \mathrm{~s}^{-1}}\right)^{-2} \\
& \times\left(\frac{P}{680 \mathrm{~s}}\right)\left(\frac{P_{\mathrm{orb}}}{41.5 \mathrm{~d}}\right)^{-1 / 2}\left(\frac{M}{1.4 M_{\odot}}\right)^{3 / 2}\left(\frac{R}{10^{6} \mathrm{~cm}}\right)^{-3} .
\end{aligned}
$$

The equivalent equation for the Illarionov \& Kompaneets (1990) model is

$$
\begin{gathered}
B \approx 2 \times 10^{14} \mathrm{G}\left(\frac{k_{w}}{0.25}\right)^{7 / 8}\left(\frac{k}{2 / 3}\right)^{-7 / 8}\left(\frac{\xi}{0.87}\right)^{-7 / 8}\left(\frac{\dot{M}_{\mathrm{eq}}}{10^{17} \mathrm{~g} / \mathrm{s}}\right)^{1 / 2}(11) \\
\left(\frac{v_{\mathrm{rel}}}{400 \mathrm{~km} \mathrm{~s}^{-1}}\right)^{-7 / 2}\left(\frac{P}{680 \mathrm{~s}}\right)^{7 / 8}\left(\frac{P_{\mathrm{orb}}}{41.5 \mathrm{~d}}\right)^{-7 / 8}\left(\frac{M}{1.4 M_{\odot}}\right)^{2}\left(\frac{R}{10^{6} \mathrm{~cm}}\right)^{-3} .
\end{gathered}
$$

Both values are in the same order of magnitude with estimates by Lipunov (1982). The strength of the magnetic field calculated under the assumption of an equilibrium period using the models for systems accreting from the persistent disk (Lovelace et al. 1999; Ghosh \& Lamb 1979) is even stronger $\left(\sim 10^{15} \mathrm{G}\right)$.

\subsection{Implications of the strong magnetic field}

As shown above, a very strong magnetic field is required to explain the long pulse period of the GX 301-2. Other authors (Li \& van den Heuvel 1999) argue that a long-period pulsating source might be a relic of magnetar evolutionary phase, when the field is strong enough to sufficiently spin the neutron star down, and demonstrate the feasibility of this scenario for 2S 0114+650. However, this does not apply to GX 301-2, since the source is in torque equilibrium and therefore the field has

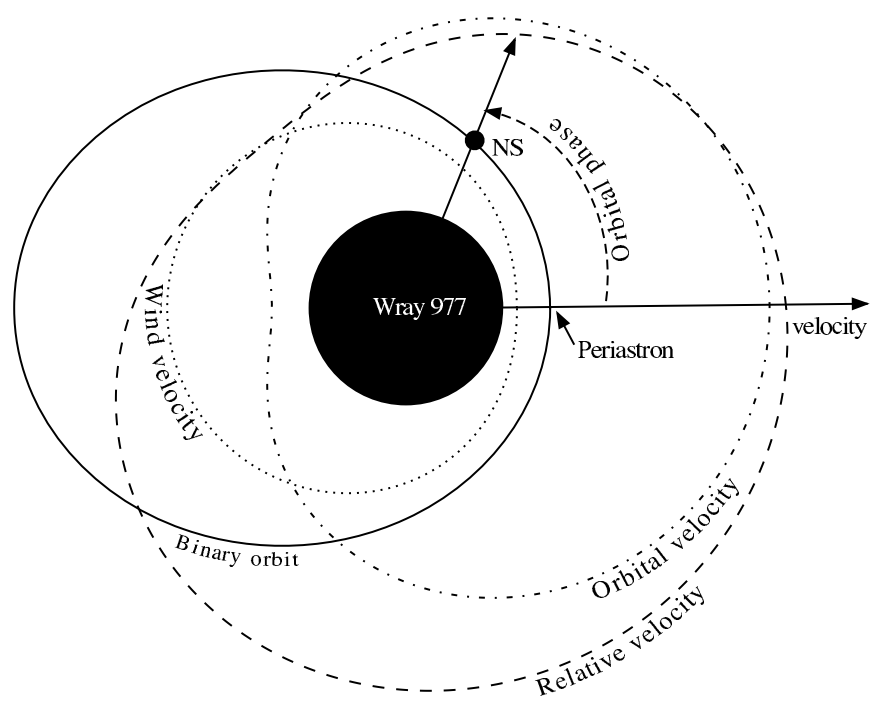

Fig. 6. Sketch of the GX 301-2 system. The relative speed of the neutron star and wind, the orbital velocity of the neutron star, and the intrinsic velocity of the wind close to the neutron star are plotted as a function of the orbital phase. Velocities are normalized to maximal relative velocity $\sim 380 \mathrm{~km} \mathrm{~s}^{-1}$, and distances to the periastron distance $\sim 1.75 \times 10^{13} \mathrm{~cm}$.

to be very strong presently, unless there are some unidentified braking torques. The observed CRSF energy, on the other hand, corresponds to a field of $B \sim 4 \times 10^{12} \mathrm{G}$, which contradicts our previous conclusion.

We suggest that it may be resolved if the line-forming region is situated far above the neutron star's surface (i.e. in the accretion column). To reconcile a surface field of $\sim 10^{14} \mathrm{G}$ with the one derived from the observed CRSF energy, one must assume that the CRSF is formed at height $H \sim R_{\mathrm{NS}}\left(\left(B_{\text {surf }} / B_{\mathrm{CRSF}}\right)^{1 / 3}-\right.$ 1) $\sim 2-3 R_{\mathrm{NS}}$ (see Fig. 8).

The accretion column rises owing to the radiation pressure when the flux from the hotspot on the neutron star surface becomes comparable to a critical flux (local Eddington luminosity). The column height increases with the accretion rate to allow the excess energy to radiate away from the side surface. The observed color temperature 4-5 keV of the GX 301-2 spectrum suggests that the accretion column is likely to form in this source. Indeed, the effective critical temperature, which corresponds to the critical flux, is $T_{\mathrm{Edd}} \sim 2 \mathrm{keV}$ for standard neutron star parameters $\left(M=1.4 M_{\odot}, R=10^{6} \mathrm{~cm}\right)$ and solar composition of the accreting matter. The observed spectrum is expected to be close to the diluted Plank spectrum $B_{E}: F_{E} \approx B_{E}\left(T_{\mathrm{c}}\right) / f_{\mathrm{c}}^{4}$, with a color temperature $T_{\mathrm{c}}=f_{\mathrm{c}} T_{\text {eff }}$ and a hardness factor $f_{\mathrm{c}} \sim 1.5-2$ because of the Compton scattering (Pavlov et al. 1991). This qualitative picture is similar in the case of Compton scattering in the strong magnetic field (Lyubarsky 1986), so the measured color temperature probably corresponds to a critical effective temperature at the neutron star surface (or at a certain height above the surface, but in this case the temperature at the surface is expected to be even higher, so the accretion column forms anyway).

It is possible to estimate the height of the accretion column, and it turns out to be compatible with the requirement mentioned above: $H \sim 2-3 R_{\mathrm{NS}}$. Indeed, the accretion column base radius can be estimated from the neutron star magnetic moment $r \approx R_{\mathrm{NS}}\left(R_{\mathrm{NS}} / R_{\mathrm{H}}\right)^{1 / 2}$ (Lipunov 1987). The magnetospheric radius is $\approx(3-30) \times 10^{8} \mathrm{~cm}$ for a magnetic field in the range 


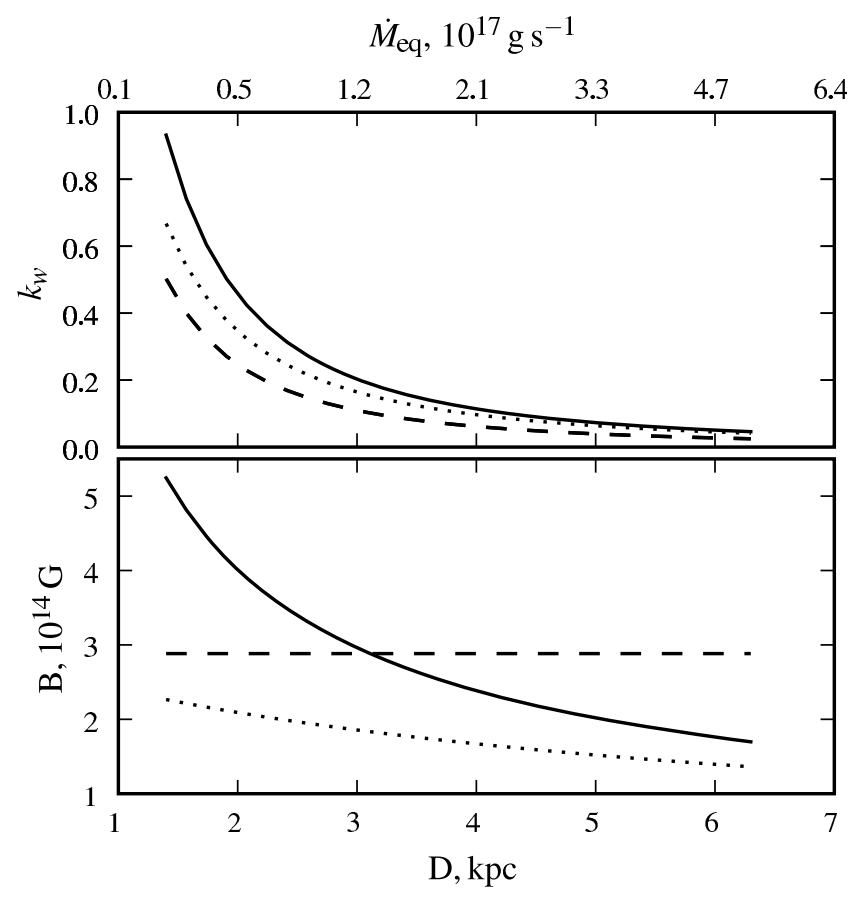

Fig. 7. Results of fitting frequency derivative - flux correlation (see Fig. 5) with Illarionov \& Kompaneets (1990) (solid), Davidson \& Ostriker (1973), Davies et al. (1979), Bisnovatyi-Kogan (1991) (dashed) and a model with both braking torques in place (dotted) depending on assumed distance, hence mean accretion rate. The required magnetic field strength B (bottom panel) depends on the efficiency of angular momentum transfer $k_{w}$ (top panel), which is constrained by the fit.

$10^{12}-10^{14} \mathrm{G}$. The corresponding radius of the column base is 200-500 m. The accretion column height may then be estimated using cylindrical geometry, and the critical effective temperature from the observed luminosity $L \approx 10^{37} \mathrm{erg} \mathrm{s}^{-1} \approx 2 \sigma_{\mathrm{SB}} T_{\mathrm{Edd}}^{4} 2 \pi r$. This simple estimate gives $H \approx 8-20 \mathrm{~km}$ (for $B=10^{12}-10^{14} \mathrm{G}$ and $\left.\dot{M}=1.2 \times 10^{17} \mathrm{~g} \mathrm{~s}^{-1}\right)$. More elaborate calculations by Basko \& Sunyaev (1976), and Lyubarsky \& Sunyaev (1988) give similar results (depending on the assumed accretion column geometry).

As shown by Basko \& Sunyaev (1976, see Fig. 4 and accompanying discussion), the amount of energy released by a unit of height of the accretion column is almost constant, so a significant part of emission comes from the outer parts of it. The contribution of the outer parts is especially important because the inner parts of the column are more easily obscured by the neutron star. It is natural to assume that X-ray emission and cyclotron line formation regions coincide, so the whole column contributes to the formation of the CRSF. The magnetic field strength and the other physical properties depend on height, so a mix of cyclotron lines coming from regions with different physical properties are observed (Nishimura 2008). The contribution of the outer parts of the column to the cyclotron line formation is especially important because the field there is weaker, so the line is observed at lower energies, which makes it easier to detect.

The formation of the pulse profile and the spectrum of a tall accretion column has not yet been understood and is beyond the scope of this work, although some characteristic features may be reckoned. The color temperature in the column increases towards the neutron star surface. The inner and hotter parts of the

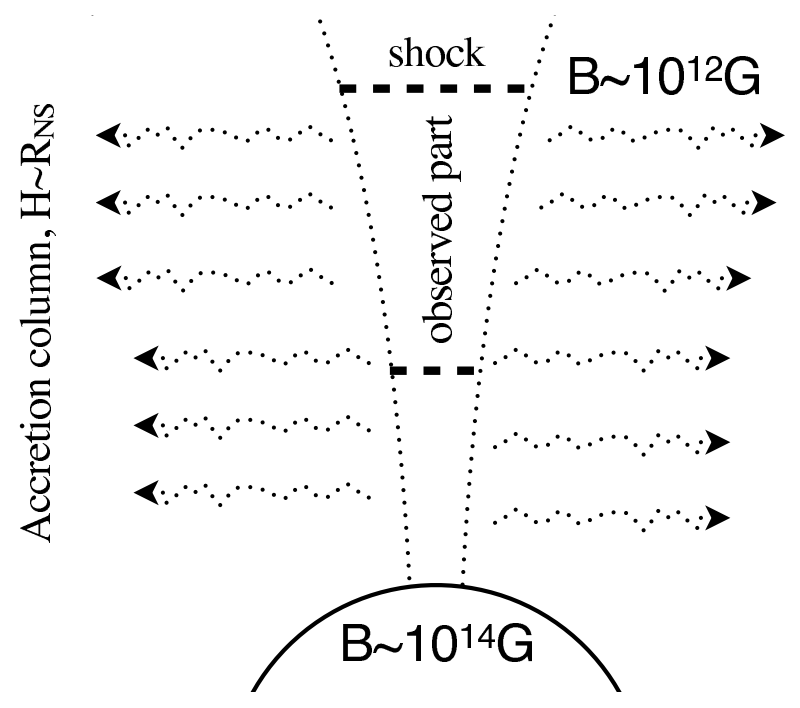

Fig. 8. Sketch of a radiation-dominated accretion column. Temperature and magnetic field strength increase towards the neutron star surface.

column are more likely to be obscured by the neutron star, so the pulse fraction increases with temperature (hence energy). For certain pulse phases, the inner parts of the column are obscured by the neutron star, and we can see only the outer, relatively cool parts, while a larger part of the column is seen at other phases. The pulse fraction is also expected to decrease with the increase in the column height and therefore with the source luminosity, which is indeed the case for GX 301-2 (Lutovinov \& Tsygankov 2009).

The magnetic field strength increases towards the neutron star, so one can expect the centroid energy of the CRSF to be anti-correlated with total column height (hence luminosity) and correlated with the effective color temperature (or the cutoff energy, which is believed to be proportional to temperature). The latter is indeed the case for GX 301-2, as reported by Kreykenbohm et al. (2004, see Table 3), while the correlation of the line energy with the luminosity is not confirmed because the cyclotron line was never detected outside of the pre-periastron flare.

When the vertical span of the observed part of the column is short, the line width is also expected to be smaller. In contrast, the line becomes wider when a larger part of the column is observed as the magnetic field increases by an order of magnitude from the column top to the bottom. This could explain the "line width - line energy" correlation reported by Kreykenbohm et al. (2004) for GX 301-2 and also by Coburn et al. (2002) for several other sources. As was concluded by Coburn et al. (2002), the observed correlation is independent of the spectral model used and is not affected by selection effects. It can therefore be considered as an intrinsic correlation between CRSF parameters. The proposed scenario with a tall accretion column explains it and complements the explanation of this correlation by Coburn et al. (2002), who attribute it to a change in the viewing angle with respect to the magnetic field (see also Meszaros 1992).

The cyclotron line formation in a tall column with temperature gradient was investigated in detail by Nishimura (2008) with similar conclusions. He considered a surface field of $\sim 10^{12} \mathrm{G}$ and about an order of magnitude less at the top of the accretion column. Both the height of the column and the range of the physical parameters within it are expected to be greater 
for a stronger surface field, but the results should be similar. Detailed modeling of the accretion column which accounts for light-bending and beaming is, however, required to clarify the expected shape, and pulse-phase dependence of the spectrum. The pulse profile shape and phase dependence of the spectrum in GX 301-2 (Kreykenbohm et al. 2004) is similar to other luminous slow-pulsating sources like Vela X-1 (Heindl et al. 2004) and 4U 1538-52 (Robba et al. 2001). Common spectral features include a correlation of CRSF energy with the width and with the cutoff energy (which characterizes the continuum temperature) in phase-resolved spectra, so the discussion above may also be relevant for these objects.

It is worth noting that the mass of the optical counterpart is very high, so one can expect that the second supernova explosion in the system may take place before the magnetic field decays significantly. Such an explosion will most likely disrupt the system and leave an isolated neutron star with a magnetar-like magnetic field and the long pulse period. The observational appearance of such an object is unclear. The strong magnetic field might power a magnetar-like emission, however, known magnetars (i.e. soft gamma-repeaters and anomalous X-ray pulsars) have much shorter pulse periods.

\section{Summary and conclusions}

In this work we studied the timing and spectral properties of GX 301-2 using the archival data of INTEGRAL and data products of CGRO BATSE and RXTE ASM. An orbital-period's secular change was detected and the pulse period history since May 2005 determined. This shows a steady spin-down trend. The apparent rate of decay of the orbital period is about an order of magnitude higher than for other known sources. We argue that this is probably caused by angular momentum loss by material expelled from the vicinity of the neutron star to the outside world. However, we cannot at this time exclude some contribution from a possible apsidal motion to the observational appearance.

Results of our spectral analysis are consistent with previous works, although we find that the spectrum is described well not only with phenomenological models, but also with a saturated comptonization model.

We discussed a possible scenario to explain the long pulse period and long spin-down trends observed despite steady accretion of matter and angular momentum onto the neutron star. We studied the balance of the torques affecting the neutron star using BATSE/DISCLA data by Bildsten et al. (1997) and find that the rotational frequency derivative is correlated with the flux. We also find that the frequency derivative is zero for the average count rate, which is a signature that the observed pulse period reflects torque equilibrium during the observations' time span. The scenario invoked by Li \& van den Heuvel (1999) to explain the long period of $2 \mathrm{~S} 0114+650$ cannot therefore be applied to GX 301-2 since the observed pulse period is close to equilibrium. We investigated several published torque models to constrain the magnetic field strength and found that all of them require the field to be $\gtrsim 10^{14} \mathrm{G}$. The magnetic field strength derived from the observed CRSF energy turns out to be $\sim 4 \times 10^{12} \mathrm{G}$, i.e. at least an order of magnitude less than from the timing. We argue that this can be explained if the line-forming region resides high up in the accretion column. We show that the accretion column as high as $\sim 25-30 \mathrm{~km}$ is expected to form in GX 301-2 in the framework of the Basko \& Sunyaev (1976) model and that it is sufficient to reconcile the very strong field at the surface with the observed cyclotron line energy. Following the scenario proposed by Nishimura (2008), we conclude that these correlations may be explained qualitatively by a simultaneous change of height and vertical span of the observed region with pulse phase if the line-forming region resides in a tall accretion column with a temperature gradient. The quantitive description of the spectrum with a model such as the one developed by Nishimura (2008) and detailed pulse profile formation modeling is, however, required to confirm this scenario.

An alternative scenario is that the long pulse period is explained by the presence of some unidentified braking torque, which is less dependent on magnetic field strength, and the CRSF pulse phase variability is attributed, as concluded by Kreykenbohm et al. (2004), to multipole field components.

Acknowledgements. We sincerely wish to thank N. Shakura, K. Postnov, and the anonymous referee for the useful comments and discussions, which helped to improve the paper. V.D., D.K., and C.F thank the DFG for financial support (grants DLR 50 OR 0702 and DLR 50 OG 0601). V.S. thanks the DFG for financial support (grant SFB/Transregio 7 "Gravitational Wave Astronomy"), and for partial support the RBRF(grant 09-02-97013-p-povolzh'e-a). We also acknowledge the support of the International Space Science Institute (Bern). This research is based on observations with INTEGRAL, an ESA project with the instruments and science data center funded by ESA member states (especially the PI countries: Denmark, France, Germany, Italy, Switzerland, Spain), the Czech Republic, and Poland, with the participation of Russia and the USA.

\section{References}

Bagot, P. 1996, A\&A, 314, 576

Basko, M. M., \& Sunyaev, R. A. 1976, MNRAS, 175, 395

Bildsten, L., Chakrabarty, D., Chiu, J., et al. 1997, ApJS, 113, 367

Bisnovatyi-Kogan, G. S. 1991, A\&A, 245, 528

Castor, J. I., Abbott, D. C., \& Klein, R. I. 1975, ApJ, 195, 157

Coburn, W., Heindl, W. A., Rothschild, R. E., et al. 2002, ApJ, 580, 394

Davidson, K., \& Ostriker, J. P. 1973, ApJ, 179, 585

Davies, R. E., Fabian, A. C., \& Pringle, J. E. 1979, MNRAS, 186, 779

de Kool, M., \& Anzer, U. 1993, MNRAS, 262, 726

Doroshenko, V., Staubert, R., Kreykenbohm, I., Santangelo, A., \& Ferrigno, C. 2008, in PoS(Integral08)115, 7th INTEGRAL Workshop

Fryxell, B. A., \& Taam, R. E. 1988, ApJ, 335, 862

Ghosh, P., \& Lamb, F. K. 1979, ApJ, 234, 296

Heindl, W. A., Rothschild, R. E., Coburn, W., et al. 2004, in X-ray Timing 2003, 714,323

Hilditch, R. W. 2001, An Introduction to Close Binary Stars, ed. R. W. Hilditch Ho, C. 1989, MNRAS, 236, 299

Illarionov, A. F., \& Kompaneets, D. A. 1990, MNRAS, 247, 219

İnam, S. Ç., \& Baykal, A. 2000, A\&A, 353, 617

Kaper, L., Lamers, H. J. G. L. M., Ruymaekers, E., van den Heuvel, E. P. J., \& Zuidervijk, E. J. 1995, A\&A, 300, 446

Kaper, L., van der Meer, A., \& Najarro, F. 2006, A\&A, 457, 595

Klochkov, D., Staubert, R., Postnov, K., Shakura, N., \& Santangelo, A. 2009, A\&A, 506, 1261

Koh, D. T., Bildsten, L., Chakrabarty, D., et al. 1997, ApJ, 479, 933

Kreykenbohm, I., Wilms, J., Coburn, W., et al. 2004, A\&A, 427, 975

La Barbera, A., Segreto, A., Santangelo, A., Kreykenbohm, I., \& Orlandini, M. 2005, A\&A, 438, 617

Larsson, S. 1996, A\&AS, 117, 197

Layton, J. T., Blondin, J. M., Owen, M. P., et al. 1998, New Astron., 3, 111

Leahy, D. A., \& Kostka, M. 2008, MNRAS, 384, 747

Li, X.-D., \& van den Heuvel, E. P. J. 1999, ApJ, 513, L45

Lipunov, V. M. 1982, AZh, 59, 888

Lipunov, V. M. 1987, The astrophysics of neutron stars

Lipunov, V. M., Börner, G., \& Wadhwa, R. S. 1992, Astrophysics of Neutron Stars, ed. V. M. Lipunov, G. Börner, \& R. S. Wadhwa

Lovelace, R. V. E., Romanova, M. M., \& Bisnovatyi-Kogan, G. S. 1999, ApJ, 514,368

Lund, N., Budtz-Jørgensen, C., Westergaard, N. J., et al. 2003, A\&A, 411, L231

Lutovinov, A. A., \& Tsygankov, S. S. 2009, Astron. Lett., 35, 433

Lyubarsky, Y. E. 1986, Astrophysics, 25, 577 
A\&A 515, A10 (2010)

Lyubarsky, Y. E., \& Sunyaev, R. A. 1988, Pis'ma Astron. Zh., 14, 920

MacQueen, J. 1967, in Proc.Berkeley Sympos. Math. Statist.

Makishima, K., \& Mihara, T. 1992, in Frontiers Science Series, Proceedings of the Yamada Conference XXVIII

Meszaros, P. 1992, High-energy radiation from magnetized neutron stars, ed. P. Meszaros

Meszaros, P., \& Nagel, W. 1985, ApJ, 299, 138

Nagase, F., Hayakawa, S., Kunieda, H., et al. 1982, ApJ, 263, 814

Nishimura, O. 2008, ApJ, 672, 1127

Orlandini, M., dal Fiume, D., Frontera, F., et al. 2000, Adv. Space Res., 25, 417

Parkes, G. E., Culhane, J. L., Mason, K. O., et al. 1980, MNRAS, 191, 547

Pavlov, G. G., Shibanov, I. A., \& Zavlin, V. E. 1991, MNRAS, 253, 193

Pravdo, S. H., \& Ghosh, P. 2001, ApJ, 554, 383
Robba, N. R., Burderi, L., Di Salvo, T., Iaria, R., \& Cusumano, G. 2001, ApJ, 562,950

Ruffert, M. 1997, A\&A, 317, 793

Ruffert, M., Arnett, D., \& Shankar, A. 1992, in BAAS, 24, BAAS, 1258

Sato, N., Nagase, F., Kawai, N., et al. 1986, ApJ, 304, 241

Shakura, N. I. 1975, Sov. Astron. Lett., 1, 223

Staubert, R., Klochkov, D., \& Wilms, J. 2009, A\&A, 500, 883

Sunyaev, R. A., \& Titarchuk, L. G. 1980, A\&A, 86, 121

Taam, R. E., \& Fryxell, B. A. 1989, ApJ, 339, 297

Ubertini, P., Lebrun, F., Di Cocco, G., et al. 2003, A\&A, 411, L131

Vedrenne, G., Roques, J.-P., Schönfelder, V., et al. 2003, A\&A, 411, L63

Watanabe, S., Sako, M., Ishida, M., et al. 2003, ApJ, 597, L37

White, N. E., Mason, K. O., Huckle, H. E., Charles, P. A., \& Sanford, P. W. 1976, ApJ, 209, L119

White, N. E., \& Swank, J. H. 1984, ApJ, 287, 856 